\title{
Review Article \\ Pluripotency of Stem Cells from Human Exfoliated Deciduous Teeth for Tissue Engineering
}

\author{
Vinicius Rosa, ${ }^{1}$ Nileshkumar Dubey, ${ }^{1}$ Intekhab Islam, ${ }^{2}$ \\ Kyung-San Min, ${ }^{3}$ and Jacques E. Nör ${ }^{4}$ \\ ${ }^{1}$ Oral Sciences, Faculty of Dentistry, National University of Singapore, Singapore 119083 \\ ${ }^{2}$ Discipline of Oral and Maxillofacial Surgery, Faculty of Dentistry, National University of Singapore, Singapore 119083 \\ ${ }^{3}$ Department of Conservative Dentistry, School of Dentistry, Chonbuk National University, Jeonju 54596, Republic of Korea \\ ${ }^{4}$ Department of Cariology, Restorative Sciences and Endodontics, School of Dentistry, University of Michigan, \\ Ann Arbor, MI 48109, USA
}

Correspondence should be addressed to Vinicius Rosa; denvr@nus.edu.sg

Received 29 January 2016; Accepted 4 May 2016

Academic Editor: Boon C. Heng

Copyright (C) 2016 Vinicius Rosa et al. This is an open access article distributed under the Creative Commons Attribution License, which permits unrestricted use, distribution, and reproduction in any medium, provided the original work is properly cited.

\begin{abstract}
Stem cells from human exfoliated deciduous teeth (SHED) are highly proliferative pluripotent cells that can be retrieved from primary teeth. Although SHED are isolated from the dental pulp, their differentiation potential is not limited to odontoblasts only. In fact, SHED can differentiate into several cell types including neurons, osteoblasts, adipocytes, and endothelial cells. The high plasticity makes SHED an interesting stem cell model for research in several biomedical areas. This review will discuss key findings about the characterization and differentiation of SHED into odontoblasts, neurons, and hormone secreting cells (e.g., hepatocytes and islet-like cell aggregates). The outcomes of the studies presented here support the multipotency of SHED and their potential to be used for tissue engineering-based therapies.
\end{abstract}

\section{Introduction}

Dental pulp is a highly vascularized connective tissue encapsulated in mineralized structure formed by enamel, dentin, and cementum. It is responsible for the homeostasis of the tooth organ and acts as a sensor to detect unhealthy stimuli [1]. The dental pulp is a source of different populations of stem cells, such as dental pulp stem cells (DPSC) in permanent teeth and stem cells from human exfoliated deciduous teeth (SHED) [2]. The latter are isolated from primary teeth, which are one of the only naturally disposable and readily accessible postnatal human tissues [3]. In fact, SHED can be isolated even from carious deciduous teeth [4]. Moreover, there are very limited ethical or legal concerns about the use of these cells, hence increasing the interest in SHED for tissue engineering research [3].

SHED were first isolated in 2003 from exfoliated human deciduous incisors. The characterization of the cells obtained in that and subsequent studies showed that SHED present positive expression for a set of embryonic stem cell markers (OCT4 and NANOG), stage-specific embryonic antigens (SSEA-3 and SSEA-4), mesenchymal stem cell markers (STRO-1 and CD146), and tumor recognition antigens (TRA-1-60 and TRA-1-81) but negative for the expression of hematopoietic markers such as CD45, CD11b/c, and HLADR [5-8].

Deciduous and permanent teeth are considerably different in regard to their development, morphological features, and physiological processes. Consequently, it is expected that SHED differ from DPSC with respect to their higher proliferation rate, sphere-like cell-cluster formation, and differentiation capacity [5, 7, 9-14]. For instance, SHED present higher levels of osteocalcin production and alkaline phosphatase activity than DPSC during osteogenic differentiation [10]. Similarly, after neurogenic stimulation, SHED present higher expression of $\beta$ III-tubulin, tyrosine hydroxylase, microtubule-associated protein 2 , and nestin compared with the DPSC [15].

SHED can cross lineage boundaries and differentiate into several types of cells, such as adipocytes, endothelial cells, and neurons $[7,12]$. SHED can also undergo osteogenic 


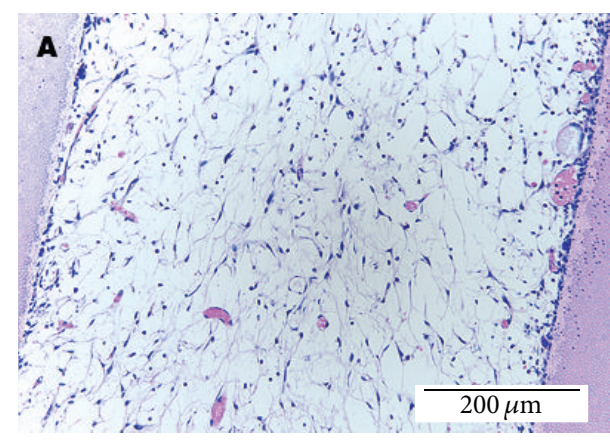

(a)

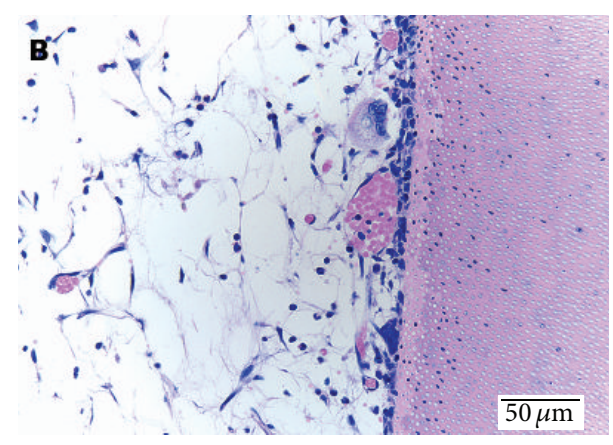

(b)

FIGURE 1: Dental pulp tissue engineered by the transplantation of SHED loaded in an injectable scaffold (a) and tooth extracted for orthodontic reasons (b). Reprinted with permission from [11] (Copyright (2011) John Wiley and Sons).

differentiation and generate bone in vivo, making these cells an interesting model for bone tissue regeneration $[7,16]$. This has been highlighted by a study where SHED mixed with $\beta$-tricalcium phosphate carrier were able to promote bone regeneration in jaw defects in swines while the carrier without cells failed to induce the same [17]. Similarly, SHED mixed with platelet-rich plasma were able to promote the formation of vascularized mature bone in defects created in the mandible of the dogs after 8 weeks [18]. Altogether, these findings expand the potential of SHED to be used for tissue engineering-based therapies involving a large number of tissues.

\section{SHED for Dental Pulp Tissue Engineering}

Despite the introduction of new materials, medicines, and tools for the clinical management of dental pulp diseases, the principles of root canal treatment have not evolved significantly from the disinfection and obturation paradigm. This well-established approach presents high rates of success in the daily clinics, but it is merely based on the substitution of organic tissues with synthetic and, in many cases, inert materials [11]. This often restricts the completion of root development in immature teeth [19]. Hence, the development of clinically approachable techniques that allow the regeneration of a functional dental pulp that is capable to deposit organized and mineralized matrix is of great interest $[3,20]$. The shift towards regenerative endodontics can lead to the rescue of tooth viability and further development of the root structure.

The first evidence that SHED could be used for dental pulp tissue regeneration was presented in the landmark paper published by Miura et al. in 2003. There, SHED were mixed with hydroxyapatite/tricalcium phosphate and implanted in the subcutaneous space of immunocompromised mice. After 8 weeks, SHED from either single or multiple colonies were capable to survive and proliferate within the scaffold and form dentin-like tissue [7].

The inherent potential of SHED to induce the formation of dental pulp in vivo gained momentum later in 2008. In that year, Cordeiro and collaborators seeded SHED in biodegradable poly-L-lactic acid-based scaffolds prepared within human tooth slices of $1 \mathrm{~mm}$ in thickness that were subsequently implanted in mice. After four weeks, a pulp-like tissue with a vascular network was formed in the space once occupied by the scaffold. In addition, cells lining the dentin surface morphologically resembled odontoblasts presenting eccentric polarized position of the nucleus on the basal part of the cell body and positive protein expression for dentin sialoprotein (DSP) [21].

The capacity of SHED to differentiate into fully functional odontoblasts capable of depositing a mineralized structure comparable to dentin in vivo was observed later. Similar to the previous study, SHED were seeded within a scaffold cast in a tooth slice and implanted subcutaneously into the dorsum of mice. 32 days after implantation, a dental pulplike tissue was centripetally formed in the pulp chamber of the tooth slice. The tissue formed with SHED had a positive expression for markers of odontoblastic differentiation such as dentin sialophosphoprotein (DSPP) and dentin matrix protein 1 (DMP-1). During the experiment, mice received periodical injections of tetracycline hydrochloride to evince the deposition of mineralized matrix. Remarkably, welldefined fluorescent lines originated from the chelation of calcium ions in the newly deposited dentin offered the evidence that SHED can differentiate into fully functional odontoblasts in vivo [12].

The translation of pulp tissue engineering to clinical setting requires the regeneration of the dental pulp within the full length of the root canal. SHED were shown to be capable to attach to the dentin walls and proliferate inside the root canals in vitro [22]. In 2013, SHED transplanted into full length root canals with injectable scaffolds were capable of proliferating within the root canal and express putative markers of odontoblastic differentiation (DSPP, DMP-1, and MEPE) after 28 days in vitro. In addition, there was the generation of a functional dental pulp in full length root canals in vivo when the roots with SHED were implanted in the subcutaneous space of mice (Figure 1). Remarkably, after 28 days from implantation, a human pulp-like tissue was occupying the majority of the space of the root canal regardless of the type of injectable scaffold used. The engineered dental pulp was capable of depositing new dentin as evinced by tetracycline injections at a rate of approximately $10 \mu \mathrm{m} /$ day [23]. The growth rate observed is within the range 
reported for primary dentin that varies according to the stage of development and age of the tooth (4 to $15 \mu \mathrm{m} /$ day) [24]. Though these findings are exciting, they are related to the ectopic tissue formation. Future studies must be performed to evaluate the capability of SHED to generate functional dental pulp in the oral environment.

\section{SHED for Neuron Tissue Engineering}

Neurodegenerative disorders are characterized by the loss or degeneration of neurons, leading to functional impairment. Although some of them, such as Parkinson and Alzheimer's disease, mainly affect older people, they are not part of the natural aging process. Parkinson disease affects 9.7 to 13.8 per 100,000 population, while Alzheimer's disease has become a major public health concern as the world's population ages $[25,26]$. In addition to these diseases, there are approximately five million people living with traumatic brain disability in the United States alone [27]. Other injuries such as stroke, peripheral nerve injury, and spinal cord injury also pose a huge burden to society. Due to the limited regenerative capacity of the nervous system, stem cell-based therapies have emerged as therapeutic options. The neural crest-cell origin of the dental pulp makes SHED an interesting cell model for neuron tissue regeneration research [28].

SHED under nonneuronal induction conditions express nestin, glial fibrillary acidic protein (GFAP), doublecortin, neuronal nuclei (NeuN), and others at both the genetic and protein levels [7, 8, 29-31]. Although SHED present neuronal traits in their undifferentiated state, these cells are prone to undergo neurogenic differentiation both in vitro and in vivo [7,32-34]. SHED treated with culture medium supplemented with epidermal growth factor (EGF) and fibroblast growth factor (FGF) presented high protein expression of neuronal markers including $\beta$ III-tubulin, glutamic acid decarboxylase (GAD), and NeuN after four weeks. Nonetheless, there was no increase in the expression of nestin, GFAP, and neurofilament M (NFM) [7]. A similar trend was observed in another study where differentiated SHED showed positive expression of both glial and neuronal markers after 21 days. Here, some of the differentiated cells presented deposits of antimyelin basic protein while the majority of cells displayed positive expression for neuronal marker $\beta$ III-tubulin. In addition, differentiated SHED presented a positive expression of apolipoprotein E (Apo E), which is present within the glia surrounding motor and sensory neurons in the peripheral nervous system. Differentiated cells were also positive for intermediate filament peripherin and Brn3a [32]. The first is a protein present in the peripheral nervous system while the latter is a transcription factor that regulates peripheral sensory neurons differentiation $[32,35]$.

The neural developmental potential of SHED in vivo was demonstrated by Miura and colleagues by injecting SHED into the dentate gyrus of the hippocampus of mice. The cells survived in the environment provided and continued to express neural markers such as NFM for more than ten days [7].

The potential of SHED to undergo neurogenic differentiation in vivo opened avenues for the use of these cells as an alternative model to treat different neuron-related conditions like focal cerebral ischemia, spinal cord injuries, Alzheimer's disease, and others.

Focal cerebral ischemia occurs when there is not enough oxygen supply due to limited blood flow to a specific region of the brain and it may lead to cerebral infarction or ischemic stroke [36, 37]. SHED can secrete compounds that positively influence the recovery of this type of lesion. Rats with focal cerebral ischemia induced by permanent middle cerebral artery occlusion presented with a significant decrease in the motor disability score when subjected to intranasal administration of supernatants from the medium used to culture SHED compared to Dulbecco's Modified Eagle's Medium (DMEM) or bone marrow stem cells conditioned DMEM used as controls. Furthermore, there was a significant decrease in the infarct volume from approximately $140 \mathrm{~mm}^{3}$ for DMEM to $50 \mathrm{~mm}^{3}$ when SHED conditioned medium was used. In addition, the animals treated with SHED conditioned media had more positive signals for neuronal nucleus, neurofilament $\mathrm{H}$, doublecortin, and rat endothelial cell antigen in the peri-infarct area when compared with the DMEM used as a control [38].

A spinal cord injury is a life-disrupting condition that triggers irreversible loss of motor and sensory functions [39]. It is estimated that up to 500,000 people suffer from spinal cord injuries per year worldwide and some severe types (e.g., $\mathrm{C} 4$ or higher lesions) may negatively affect breathing since the lesion affects the autonomic control system [40]. SHED have remarkable neuroregenerative activity and can promote functional recovery after spinal cord injury. Rats that received SHED within the space created by the complete transection at the 9th-11th thoracic vertebral levels exhibited higher scores in the Basso, Beattie, and Bresnahan locomotor rating scale compared to those transplanted with bone marrow stromal cells or fibroblasts. The rescue of hind limb locomotor function was also higher in the rats that received SHED. These animals were able to move three joints of hind limb coordinately and walk, while the treatment with bone marrow stromal cells allowed only subtle movements of one or two joints. The improvements observed with SHED can be related to their differentiation into mature oligodendrocytes that replace lost cells and regeneration of cleaved axons (Figure 2) [8].

The enhanced regeneration observed with SHED not only applies to lesions in the central nervous system, but also was observed in peripheral nerve injuries. It was possible to promote the regeneration of sciatic nerve defects in rats by treating the lesions with SHED conditioned media. The axon density and number of regenerated myelinated fibers observed in the group treated with SHED conditioned media were similar to the autograft used as a control [41].

These promising results pertaining to the neuron differentiation potential of SHED in several in vitro settings and animal models increase the interest of using these cells as an alternative for treating different neuronal diseases and injuries.

\section{SHED Differentiation towards Hormone Secreting Cells}

In addition to their therapeutic use in dental pulp and neuroregeneration, SHED also have the potential to be used in the treatment of hepatic diseases and diabetes. 
(a)

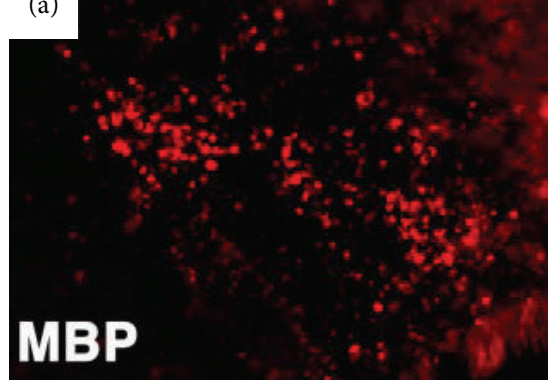

(d)

\section{APC}

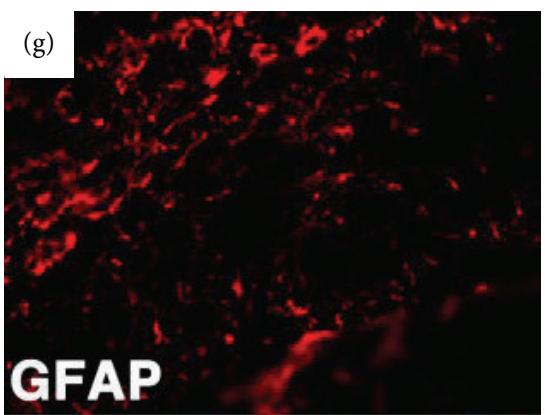

(j)

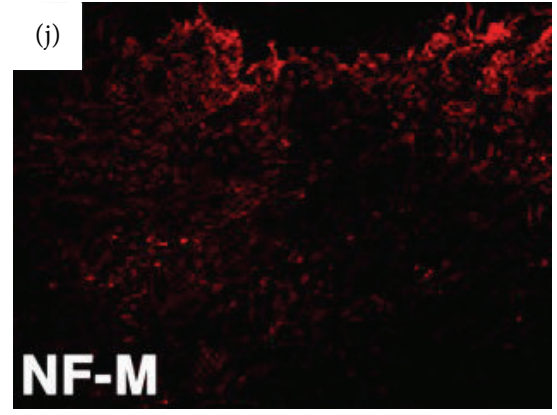

(b)

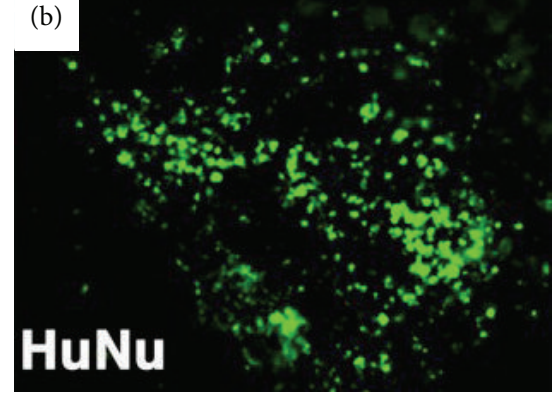

(e)

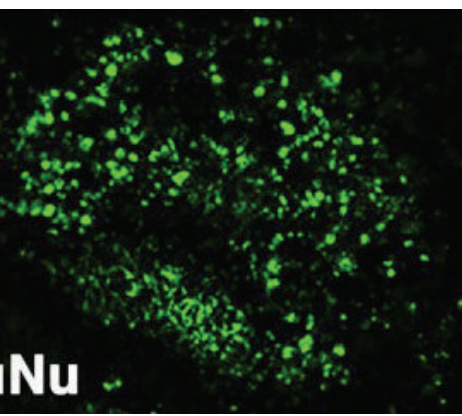

(h)

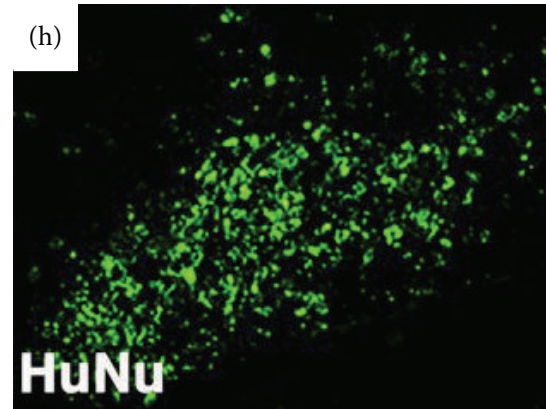

(k)

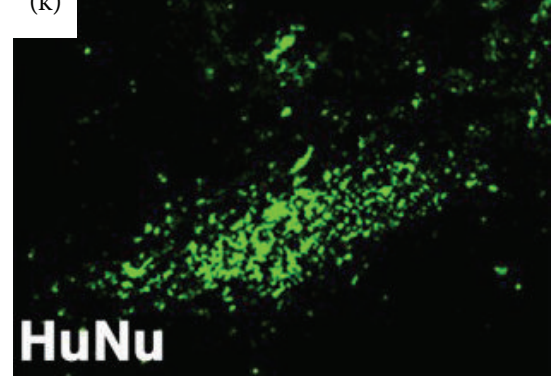

) (c)

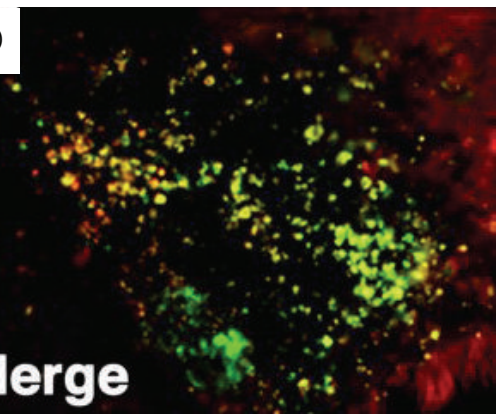

(f)

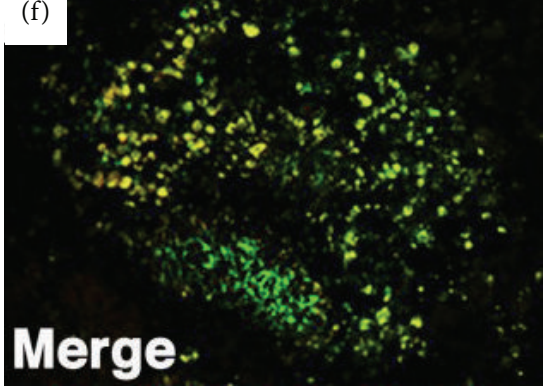

(i)

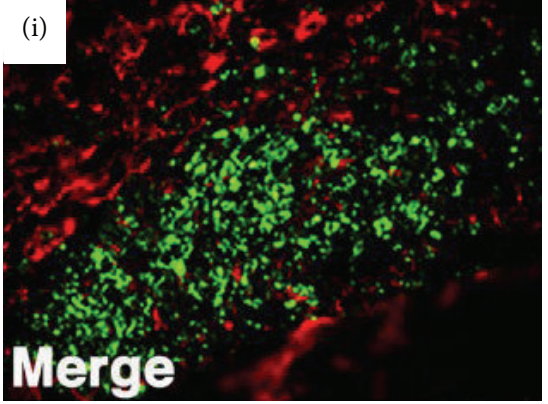

(1)

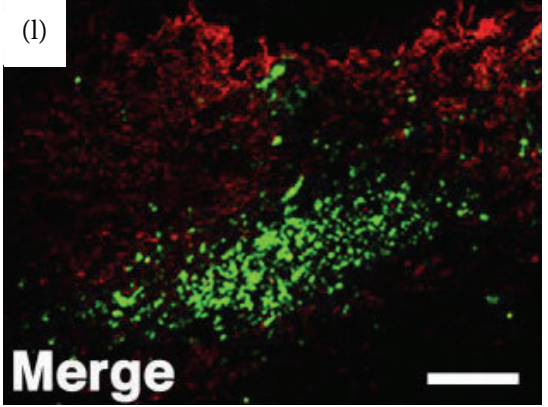

FIGURE 2: Immunohistochemical staining showed that SHED transplanted into transected spinal cord differentiated into mature oligodendrocytes [8] (Copyright (2012) American Society for Clinical Investigation.)

Organ transplantation may be the choice of treatment for patients suffering from fatal liver conditions such as cirrhosis and hepatocellular carcinoma. However, the scarcity of donors encourages the development of therapeutic alternatives. Recent studies provide evidence that SHED can differentiate into hepatic lineage cells [42-45]. Under proper stimulation, SHED express a set of hepatic markers such as hepatic nuclear factor- $4 \alpha, \alpha$-fetoprotein, and insulin-like growth factor-1. Remarkably, $90 \%$ of the hepatocytes obtained were positive for the expression of albumin. In addition, there were significant increases in the concentration of urea in the media and amount of cytoplasmic glycogen storage within the cells after differentiation [43]. The level of differentiation can be further enhanced by addition of liquorice or angelica extracts in the culture medium [46] or by treating with hydrogen sulfide $\left(\mathrm{H}_{2} \mathrm{~S}\right)$ [47]. The transplantation of SHED into the liver of mice with fibrosis induced by carbon tetrachloride showed that the cells can participate in the hepatic recovery via both direct (tissue replacement) and indirect (antifibrotic and antiinflammatory effects) integration [48].

Diabetes is one of the most common chronic endocrinal diseases associated with destruction and dysfunction of 
pancreatic $\beta$-cells. As SHED can differentiate in vitro into islet-like cell aggregates (ICA), they emerge as an alternative towards cell replacement therapy for diabetes $[49,50]$. It has been shown that the SHED-originated ICA can release insulin and C-peptide in a glucose-dependent manner in vitro [49]. The incubation of MIN6 (mouse pancreatic $\beta$-cell line) with SHED conditioned medium enhanced insulin secretion in a glucose concentration-dependent manner [51]. Mice with diabetes induced by streptozotocin which were transplanted with islet-like cells derived from SHED reversed the diabetes and restored the normoglycemia after three to four weeks [50].

\section{Conclusion}

As SHED can be obtained from naturally "disposable" tissues without significant morbidity to host and with limited ethical concern, they present another opportunity for dentistry to contribute to the development of tissue engineering. Several studies offer the evidence that SHED can differentiate into odontoblasts, neurons, hepatocytes, endothelial cells, $\beta$-cells, and others. This wide variety of cell types creates a plethora of opportunities for the use of SHED in tissue regeneration processes. Nonetheless, there is still the need to deepen the understanding of the mechanisms underlying the differentiation processes before SHED-based therapies can become a clinical reality.

\section{Competing Interests}

The authors declare that they have no competing interests.

\section{Acknowledgments}

Vinicius Rosa was supported by grants from the National Medical Research Council, Singapore (NMRC/CNIG/1107/2013), and National University Health System (NUHSRO/2014/017/ B2B/02). Kyung-San Min acknowledges Basic Science Research Program through the National Research Foundation of Korea funded by the Ministry of Education, Science and Technology (2013-R1A1A4A01006389).

\section{References}

[1] A. Nanci, Ten Cate's Oral Histology-Pageburst on VitalSource: Development, Structure, and Function, Elsevier Health Sciences, Philadelphia, Pa, USA, 2007.

[2] V. Rosa, "What and where are the stem cells for dentistry?" Singapore Dental Journal, vol. 34, no. 1, pp. 13-18, 2013.

[3] V. Rosa, T. M. Botero, and J. E. Nör, "Regenerative endodontics in light of the stem cell paradigm," International Dental Journal, vol. 61, no. 1, pp. 23-28, 2011.

[4] S. B. Werle, D. Lindemann, D. Steffens et al., "Carious deciduous teeth are a potential source for dental pulp stem cells," Clinical Oral Investigations, vol. 20, no. 1, pp. 75-81, 2016.

[5] S. Gronthos, M. Mankani, J. Brahim, P. G. Robey, and S. Shi, "Postnatal human dental pulp stem cells (DPSCs) in vitro and in vivo," Proceedings of the National Academy of Sciences of the United States of America, vol. 97, no. 25, pp. 13625-13630, 2000.
[6] I. Kerkis, A. Kerkis, D. Dozortsev et al., "Isolation and characterization of a population of immature dental pulp stem cells expressing OCT-4 and other embryonic stem cell markers," Cells Tissues Organs, vol. 184, no. 3-4, pp. 105-116, 2006.

[7] M. Miura, S. Gronthos, M. Zhao et al., "SHED: stem cells from human exfoliated deciduous teeth," Proceedings of the National Academy of Sciences of the United States of America, vol. 100, no. 10, pp. 5807-5812, 2003.

[8] K. Sakai, A. Yamamoto, K. Matsubara et al., "Human dental pulp-derived stem cells promote locomotor recovery after complete transection of the rat spinal cord by multiple neuroregenerative mechanisms," Journal of Clinical Investigation, vol. 122, no. 1, pp. 80-90, 2012.

[9] S. Bojic, V. Volarevic, B. Ljujic, and M. Stojkovic, "Dental stem cells-characteristics and potential," Histology and Histopathology, vol. 29, no. 6, pp. 699-706, 2014.

[10] N. Koyama, Y. Okubo, K. Nakao, and K. Bessho, "Evaluation of pluripotency in human dental pulp cells," Journal of Oral and Maxillofacial Surgery, vol. 67, no. 3, pp. 501-506, 2009.

[11] V. Rosa, T. M. Botero, and J. E. Nör, "Regenerative endodontics in light of the stem cell paradigm," International Dental Journal, vol. 61, supplement 1, pp. 23-28, 2011.

[12] V. T. Sakai, Z. Zhang, Z. Dong et al., "SHED differentiate into functional odontoblasts and endothelium," Journal of Dental Research, vol. 89, no. 8, pp. 791-796, 2010.

[13] S. Shi, P. G. Robey, and S. Gronthos, "Comparison of human dental pulp and bone marrow stromal stem cells by cDNA microarray analysis," Bone, vol. 29, no. 6, pp. 532-539, 2001.

[14] S. Gronthos, J. Brahim, W. Li et al., "Stem cell properties of human dental pulp stem cells," Journal of Dental Research, vol. 81, no. 8, pp. 531-535, 2002.

[15] X. Feng, J. Xing, G. Feng et al., "Age-dependent impaired neurogenic differentiation capacity of dental stem cell is associated with wnt/ $\beta$-catenin signaling," Cellular and Molecular Neurobiology, vol. 33, no. 8, pp. 1023-1031, 2013.

[16] B. M. Seo, W. Sonoyama, T. Yamaza et al., "SHED repair criticalsize calvarial defects in mice," Oral Diseases, vol. 14, no. 5, pp. 428-434, 2008.

[17] Y. Zheng, Y. Liu, C. M. Zhang et al., "Stem cells from deciduous tooth repair mandibular defect in swine," Journal of Dental Research, vol. 88, no. 3, pp. 249-254, 2009.

[18] Y. Yamada, K. Ito, S. Nakamura, M. Ueda, and T. Nagasaka, "Promising cell-based therapy for bone regeneration using stem cells from deciduous teeth, dental pulp, and bone marrow," Cell Transplantation, vol. 20, no. 7, pp. 1003-1013, 2011.

[19] J. E. Nör, "Tooth regeneration in operative dentistry," Operative Dentistry, vol. 31, no. 6, pp. 633-642, 2006.

[20] M. T. P. Albuquerque, M. C. Valera, M. Nakashima, J. E. Nör, and M. C. Bottino, "Tissue-engineering-based strategies for regenerative endodontics," Journal of Dental Research, vol. 93, no. 12, pp. 1222-1231, 2014.

[21] M. M. Cordeiro, Z. Dong, T. Kaneko et al., "Dental pulp tissue engineering with stem cells from exfoliated deciduous teeth," Journal of Endodontics, vol. 34, no. 8, pp. 962-969, 2008.

[22] E. L. Gotlieb, P. E. Murray, K. M. Namerow, S. Kuttler, and F. Garcia-Godoy, "An ultrastructural investigation of tissueengineered pulp constructs implanted within endodontically treated teeth," Journal of the American Dental Association, vol. 139, no. 4, pp. 457-465, 2008.

[23] V. Rosa, Z. Zhang, R. H. M. Grande, and J. E. Nör, "Dental pulp tissue engineering in full-length human root canals," Journal of Dental Research, vol. 92, no. 11, pp. 970-975, 2013. 
[24] K. Kawasaki, S. Tanaka, and T. Ishikawa, "On the incremental lines in human dentine as revealed by tetracycline labelling," Journal of Anatomy, vol. 123, no. 2, pp. 427-436, 1977.

[25] B. Duthey, "Background paper 6.11: Alzheimer disease and other dementias," A Public Health Approach to Innovation, pp. 1-74, 2013.

[26] C. Marras and C. M. Tanner, "Epidemiology of Parkinson's disease," in Movement Disorders, Neurologic Principles and Practic, R. L. Watts and W. C. Koller, Eds., pp. 177-196, McGraw Hill, New York, NY, USA, 2nd edition, 2004.

[27] D. F. Kelly and D. P. Becker, "Advances in management of neurosurgical trauma: USA and Canada," World Journal of Surgery, vol. 25, no. 9, pp. 1179-1185, 2001.

[28] S. Nakamura, Y. Yamada, W. Katagiri, T. Sugito, K. Ito, and M. Ueda, "Stem cell proliferation pathways comparison between human exfoliated deciduous teeth and dental pulp stem cells by gene expression profile from promising dental pulp," Journal of Endodontics, vol. 35, no. 11, pp. 1536-1542, 2009.

[29] Y. Chai, X. Jiang, Y. Ito et al., "Fate of the mammalian cranial neural crest during tooth and mandibular morphogenesis," Development, vol. 127, no. 8, pp. 1671-1679, 2000.

[30] F. Santagati and F. M. Rijli, "Cranial neural crest and the building of the vertebrate head," Nature Reviews Neuroscience, vol. 4, no. 10, pp. 806-818, 2003.

[31] D. Majumdar, M. Kanafi, R. Bhonde et al., "Differential neuronal plasticity ofdental pulp stem cells from exfoliated deciduous and permanent teeth towards dopaminergic neurons," Journal of Cellular Physiology, 2016.

[32] A. Jarmalavičiute, V. Tunaitis, E. Strainiene et al., "A new experimental model for neuronal and glial differentiation using stem cells derived from human exfoliated deciduous teeth," Journal of Molecular Neuroscience, vol. 51, no. 2, pp. 307-317, 2013.

[33] A. Esmaeili, S. Alifarja, N. Nourbakhsh, and A. Talebi, "Messenger RNA expression patterns of neurotrophins during transdifferentiation of stem cells from human-exfoliated deciduous teeth into neural-like cells," Avicenna Journal of Medical Biotechnology, vol. 6, no. 1, pp. 21-26, 2014.

[34] N. Nourbakhsh, M. Soleimani, Z. Taghipour et al., "Induced in vitro differentiation of neural-like cells from human exfoliated deciduous teeth-derived stem cells," International Journal of Developmental Biology, vol. 55, no. 2, pp. 189-195, 2011.

[35] W. J. Pavan and D. W. Raible, "Specification of neural crest into sensory neuron and melanocyte lineages," Developmental Biology, vol. 366, no. 1, pp. 55-63, 2012.

[36] N. S. Russek and M. B. Jensen, "Histological quantification of brain tissue inflammatory cell infiltration after focal cerebral infarction: a systematic review," International Journal of Neuroscience, vol. 124, no. 3, pp. 160-165, 2014.

[37] M. Balkaya, J. M. Kröber, A. Rex, and M. Endres, "Assessing post-stroke behavior in mouse models of focal ischemia," Journal of Cerebral Blood Flow and Metabolism, vol. 33, no. 3, pp. 330-338, 2013.

[38] T. Inoue, M. Sugiyama, H. Hattori, H. Wakita, T. Wakabayashi, and M. Ueda, "Stem cells from human exfoliated deciduous tooth-derived conditioned medium enhance recovery of focal cerebral ischemia in rats," Tissue Engineering-Part A, vol. 19, no. 1-2, pp. 24-29, 2013.

[39] D. Cantinieaux, R. Quertainmont, S. Blacher et al., "Conditioned medium from bone marrow-derived mesenchymal stem cells improves recovery after spinal cord injury in rats: an original strategy to avoid cell transplantation," PLoS ONE, vol. 8, no. 8, Article ID e69515, 2013.

[40] S. Lukersmith, International Perspectives on Spinal Cord Injury, 2013.

[41] Y. Sugimura-Wakayama, W. Katagiri, M. Osugi et al., "Peripheral nerve regeneration by secretomes of stem cells from human exfoliated deciduous teeth," Stem Cells and Development, vol. 24, no. 22, pp. 2687-2699, 2015.

[42] N. Ishkitiev, K. Yaegaki, T. Imai et al., "Novel management of acute or secondary biliary liver conditions using hepatically differentiated human dental pulp cells," Tissue Engineering Part A, vol. 21, no. 3-4, pp. 586-593, 2015.

[43] N. Ishkitiev, K. Yaegaki, T. Imai et al., "High-purity hepatic lineage differentiated from dental pulp stem cells in serum-free medium," Journal of Endodontics, vol. 38, no. 4, pp. 475-480, 2012.

[44] P. Vasanthan, N. Gnanasegaran, V. Govindasamy et al., "Comparison of fetal bovine serum and human platelet lysate in cultivation and differentiation of dental pulp stem cells into hepatic lineage cells," Biochemical Engineering Journal, vol. 88, pp. 142-153, 2014.

[45] N. Ishkitiev, K. Yaegaki, B. Calenic et al., "Deciduous and permanent dental pulp mesenchymal cells acquire hepatic morphologic and functional features in vitro," Journal of Endodontics, vol. 36, no. 3, pp. 469-474, 2010.

[46] W.-T. Su and X.-W. Chen, "Stem cells from human exfoliated deciduous teeth differentiate into functional hepatocyte-like cells by herbal medicine," Bio-Medical Materials and Engineering, vol. 24, no. 6, pp. 2243-2247, 2014.

[47] M. Okada, N. Ishkitiev, K. Yaegaki et al., "Hydrogen sulphide increases hepatic differentiation of human tooth pulp stem cells compared with human bone marrow stem cells," International Endodontic Journal, vol. 47, no. 12, pp. 1142-1150, 2012.

[48] T. Yamaza, F. S. Alatas, R. Yuniartha et al., "In vivo hepatogenic capacity and therapeutic potential of stem cells from human exfoliated deciduous teeth in liver fibrosis in mice," Stem Cell Research and Therapy, vol. 6, no. 1, article 171, 2015.

[49] V. Govindasamy, V. S. Ronald, A. N. Abdullah et al., "Differentiation of dental pulp stem cells into islet-like aggregates," Journal of Dental Research, vol. 90, no. 5, pp. 646-652, 2011.

[50] M. M. Kanafi, Y. B. Rajeshwari, S. Gupta et al., "Transplantation of islet-like cell clusters derived from human dental pulp stem cells restores normoglycemia in diabetic mice," Cytotherapy, vol. 15, no. 10, pp. 1228-1236, 2013.

[51] T. Izumoto-Akita, S. Tsunekawa, A. Yamamoto et al., "Secreted factors from dental pulp stem cells improve glucose intolerance in streptozotocin-induced diabetic mice by increasing pancreatic $\beta$-cell function," BMJ Open Diabetes Research \& Care, vol. 3, no. 1, Article ID e000128, 2015. 

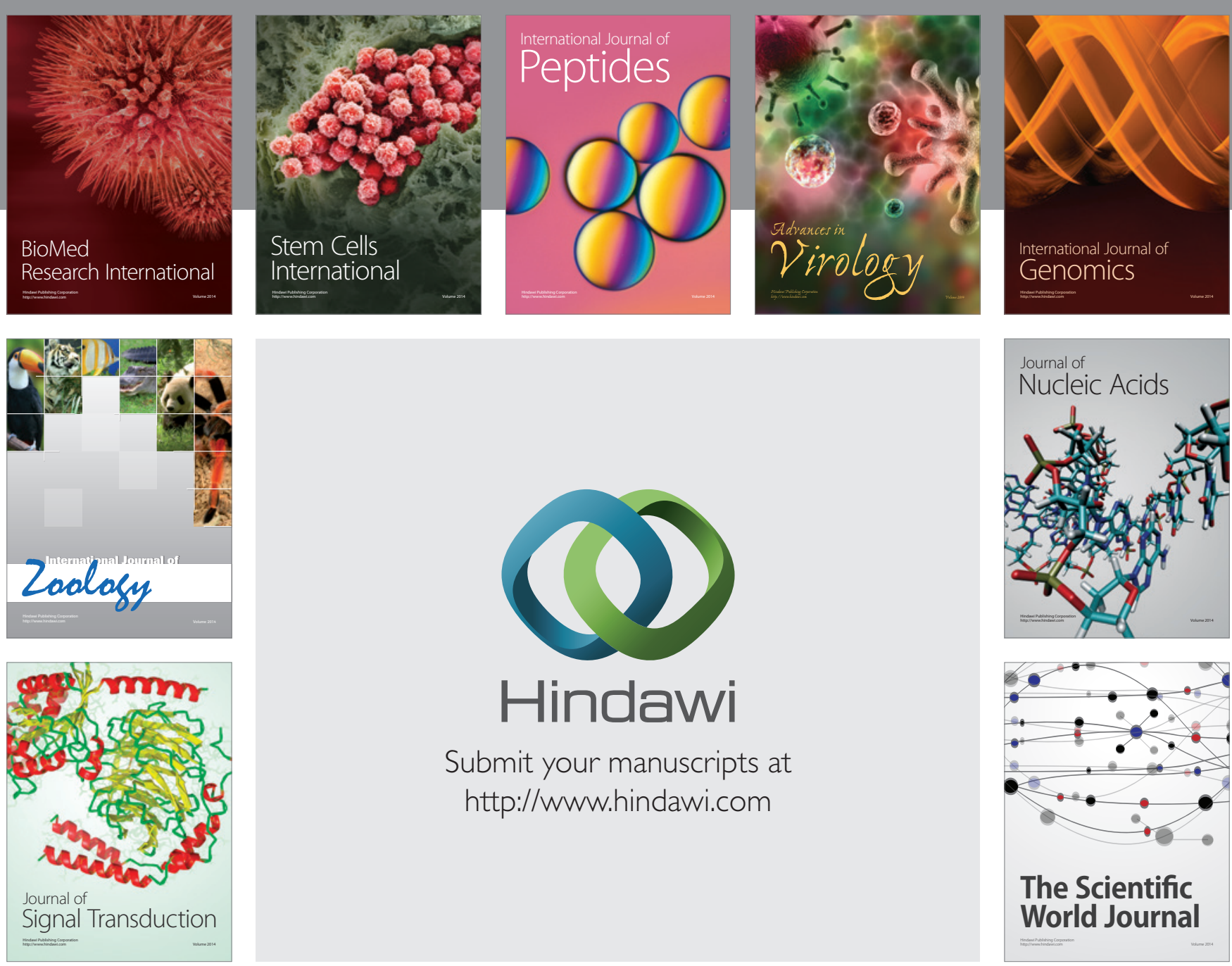

Submit your manuscripts at

http://www.hindawi.com
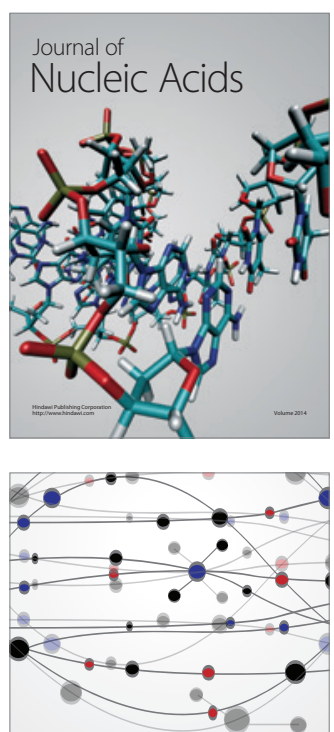

The Scientific World Journal
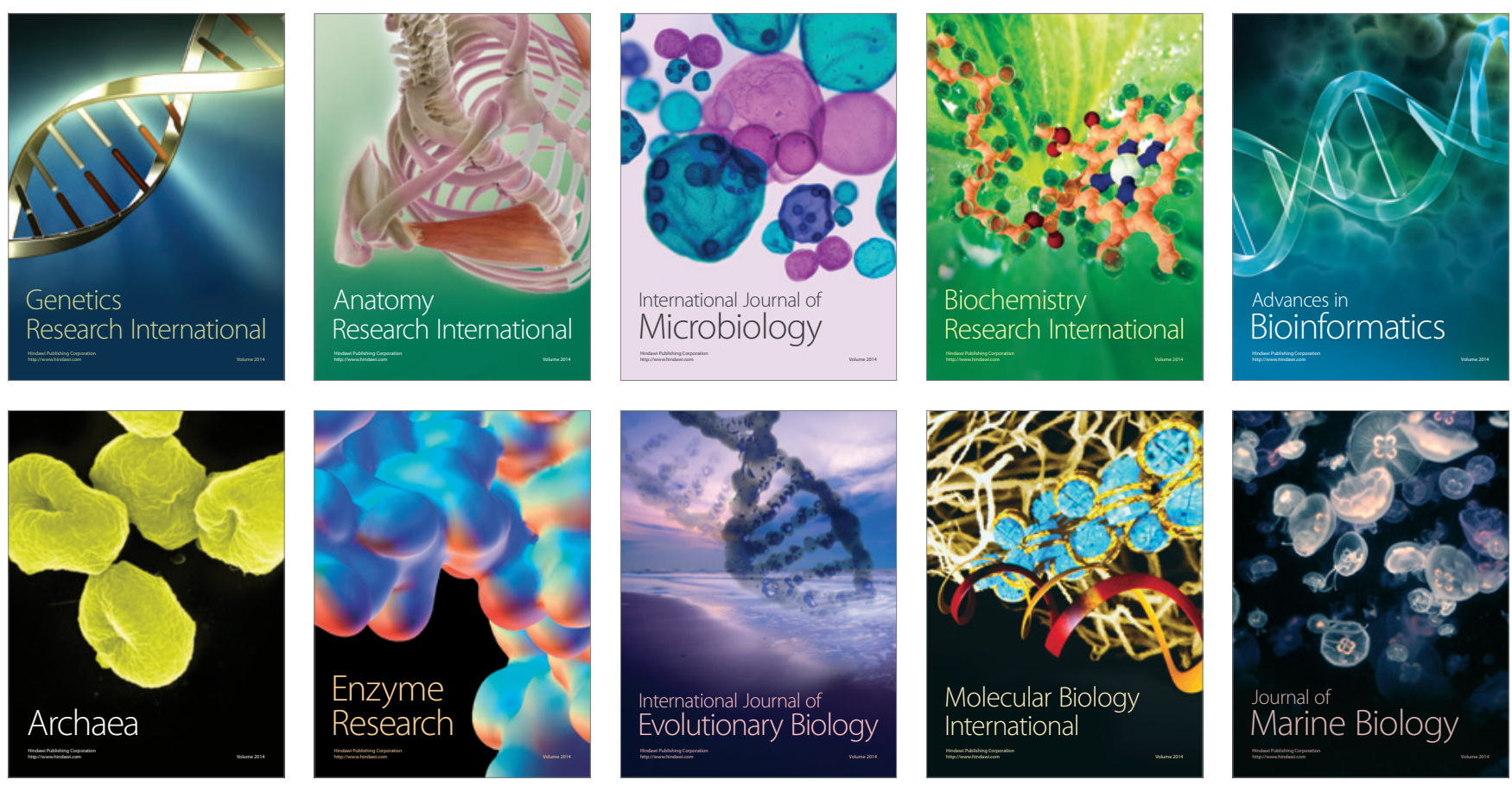